\title{
Collisions in Classical Mechanics in Terms of Mass-Momentum "Vectors" with Galilean Transformations
}

\author{
Akihiro Ogura \\ Laboratory of Physics, Nihon University, Matsudo, Japan \\ Email: ogura.akihiro@nihon-u.ac.jp
}

How to cite this paper: Ogura, A. (2020) Collisions in Classical Mechanics in Terms of Mass-Momentum "Vectors" with Galilean Transformations. World Journal of Mechanics, 10, 154-165.

https://doi.org/10.4236/wjm.2020.1010011

Received: August 28, 2020

Accepted: October 9, 2020

Published: October 12, 2020

Copyright $\odot 2020$ by author(s) and Scientific Research Publishing Inc. This work is licensed under the Creative Commons Attribution International License (CC BY 4.0).

http://creativecommons.org/licenses/by/4.0/

\begin{abstract}
We present the usefulness of mass-momentum "vectors" to analyze the collision problems in classical mechanics for both one and two dimensions with Galilean transformations. The Galilean transformations connect the massmomentum "vectors" in the center-of-mass and the laboratory systems. We show that just moving the two systems to and fro, we obtain the final states in the laboratory systems. This gives a simple way of obtaining them, in contrast with the usual way in which we have to solve the simultaneous equations. For one dimensional collision, the coefficient of restitution is introduced in the center-of-mass system. This clearly shows the meaning of the coefficient of restitution. For two dimensional collisions, we only discuss the elastic collision case. We also discuss the case of which the target particle is at rest before the collision. In addition to this, we discuss the case of which the two particles have the same masses.
\end{abstract}

\section{Keywords}

Collision, Newtonian Mechanics, Mass-Momentum Space, Galilean Transformation

\section{Introduction}

Collisions of the interacting particles have fundamental importance in physics. To concerning collision problems in classical mechanics, it is customary that the initial states, for example the mass and momenta, are given. Then, we want to find the final states, especially, momenta and energies after the collision. To obtain the final states, we have to solve the simultaneous equations of the momentum-conservation law and the definition of the coefficient of restitution [1]. Be- 
sides, it is also important to obtain the relation between the laboratory and the center-of-mass systems. It is complicated to understand the collision problems in classical mechanics.

The purpose of this paper is that we resolve the complexity of the collision problems and we show a new looking at them. In recent years, the mass-momentum diagram is used to analyze the collision problems in Newtonian mechanics [2] [3]. There, the mass-momentum "vectors" are introduced. These diagrams show the whole story of the collision problems in both the laboratory and the center-of-mass systems. However, the discussion of the paper [3] still stuck to the simultaneous equations. This paper shows that we never solve them for obtaining the final states. This diagrammatic approach is also used for two-dimensional collision problems [4] [5] [6]. There, we see the collision problems on the two-dimensional momentum space. This paper also gives the theoretical background of them.

Now consider two reference frames $K$ and $K^{\prime}$. We assume that the frame $K^{\prime}$ moves in the $X$-direction at speed $V$ with respect to $K$. And let us assume the origins $O$ and $O^{\prime}$ of the two reference frames coincide with time $t=0$. An event that occurs at some point is observed from both frames, which are characterized by a set of coordinates $(t, x, y, z)$ and $\left(t^{\prime}, x^{\prime}, y^{\prime}, z^{\prime}\right)$. The Galilean transformations give the relation between two coordinates and it is described by

$$
\left(\begin{array}{l}
t^{\prime} \\
x^{\prime} \\
y^{\prime} \\
z^{\prime}
\end{array}\right)=\left(\begin{array}{cccc}
1 & 0 & 0 & 0 \\
-V & 1 & 0 & 0 \\
0 & 0 & 1 & 0 \\
0 & 0 & 0 & 1
\end{array}\right)\left(\begin{array}{l}
t \\
x \\
y \\
z
\end{array}\right) .
$$

The inverse transformation is given by just putting $-V$ to $V$ in Equation (1). In the following paper, we designate the frame $K$ as the laboratory system, while $K^{\prime}$ as the center-of-mass system. Accordingly, the velocity $V$ describes the velocity of the center-of-mass.

In this article, we show that the collision problems are solved by using mass-momentum "vectors" $\left(m, p_{x}, p_{y}, p_{z}\right)$ and this Galilean transformation. Our strategy and notations are pictorially stated in Figure 1. In the introductory textbooks of physics [1], we have to calculate the simultaneous equations of momentum-conservation with energy-conservation or the definition of the coefficient of restitution in order to obtain the final states. See the dashed arrow in Figure 1. However, we propose that we make a detour through the center-of-mass system for obtaining the final states. It is applicable to both one and two dimensional collisions. Our strategy is as follows.

1) By the Galilean inverse transformation, we obtain the velocity $V$ of the center-of-mass in terms of masses $\left(m_{A}, m_{B}\right)$ and momenta $\left(p_{A}, p_{B}\right)$ in the laboratory system before the collision. The velocity $V$ is conserved throughout the collision.

2) By the Galilean transformation, we obtain the momenta $\left(p_{A}^{*}, p_{B}^{*}\right)$ in the center-of-mass system before the collision. See the strategy 2 in Figure 1 . We 


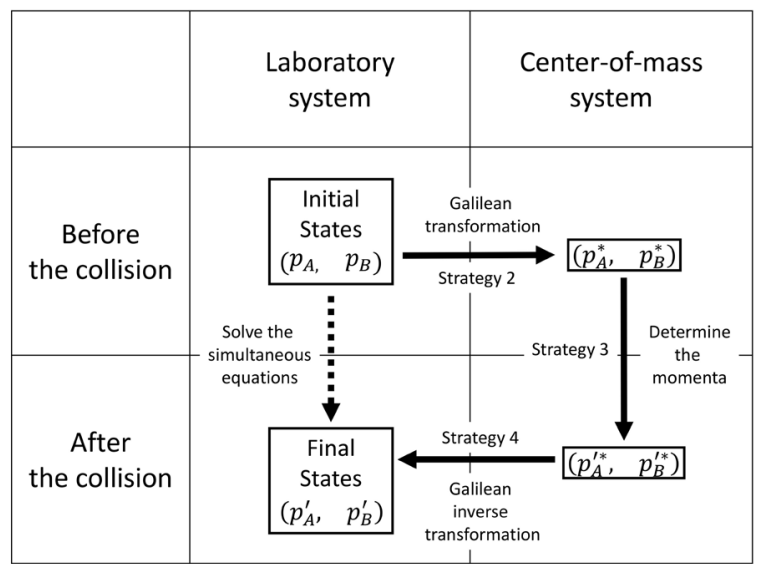

Figure 1. The usual approach to the collision problems is along the dashed arrow. The strategy in this article is on the detour of the solid arrows. The asterisks are attached to the variables in the center-of-mass system, while the primes are attached to the variables after the collision.

attach the asterisk for the variables in the center-of-mass system. In this frame, two particles make a head on collision with the momentum which have the same magnitude $p^{*}$.

3) We determine the momenta $\left(p_{A}^{\prime *}, p_{B}^{\prime *}\right)$ in the center-of-mass system after the collision. See the strategy 3 in Figure 1. We also attach the prime for the variables after the collision. In this frame, two particles move in the opposite direction after the collision with the same magnitude of momentum. We introduce the coefficient of restitution $e$ for one dimensional collisions and the collision angle $\theta^{*}$ of the incident particle for two dimensional elastic collisions.

4) By the Galilean inverse transformation, we obtain the momenta $\left(p_{A}^{\prime}, p_{B}^{\prime}\right)$ in the laboratory system after the collision. See the strategy 4 in Figure 1. Finally, we reach the final states. We never solve the simultaneous equations in contrast with the usual treatment of the collision problems.

$5)$ Let us consider the two special cases. One is that the target particle is at rest $\left(p_{B}=0\right)$ in the laboratory system before the collision.

6) The other is that, in addition to the case $\left(p_{B}=0\right)$ above, two particles have equal masses $\left(m_{A}=m_{B}\right)$.

This paper is organized in the following way. In Section 2, we discuss one dimensional collision. The role of the coefficient of restitution $e$ is clearly stated. We also discuss the case in which the target particle is at rest before the collision and two particles have equal masses. In Section 3, we turn to the two dimensional elastic collision. We introduce the collision angle $\theta^{*}$ of the outgoing particle after the collision. We show the theoretical background for the diagrammatic approach [4] [5] [6]. Section 4 is devoted to a summary.

\section{Collisions in One Dimension}

Let us discuss the one dimensional collisions. The motions of the particles are restricted in the $x$-direction. Therefore, the $y$ - and $z$-components of the mo- 
mentum are zero. The diagrammatic technique of this section is fully discussed in [3]

\subsection{Velocity of Center-of-Mass System}

We discuss the strategy 1 in the Introduction. Consider the Galilean inverse transformation with the whole two body system,

$$
\left(\begin{array}{c}
m_{A}+m_{B} \\
p_{A}+p_{B} \\
0 \\
0
\end{array}\right)=\left(\begin{array}{cccc}
1 & 0 & 0 & 0 \\
V & 1 & 0 & 0 \\
0 & 0 & 1 & 0 \\
0 & 0 & 0 & 1
\end{array}\right)\left(\begin{array}{c}
m_{A}+m_{B} \\
p_{A}^{*}+p_{B}^{*} \\
0 \\
0
\end{array}\right)
$$

where, $p_{A}^{*}+p_{B}^{*}=0$ is the definition of the center-of-mass system. From the second row of the matrix, we obtain the velocity of the center-of-mass,

$$
V=\frac{p_{A}+p_{B}}{m_{A}+m_{B}}
$$

which is conserved throughout the collision because of the conservation law of mass and momentum in Newtonian mechanics.

\subsection{Momentum in the Center-of-Mass System before the Collision}

We discuss the strategy 2 in the Introduction. Concerning the Galilean transformation for each particle,

$$
\left(\begin{array}{c}
m_{A} \\
p_{A}^{*} \\
0 \\
0
\end{array}\right)=\left(\begin{array}{cccc}
1 & 0 & 0 & 0 \\
-V & 1 & 0 & 0 \\
0 & 0 & 1 & 0 \\
0 & 0 & 0 & 1
\end{array}\right)\left(\begin{array}{c}
m_{A} \\
p_{A} \\
0 \\
0
\end{array}\right), \quad\left(\begin{array}{c}
m_{B} \\
p_{B}^{*} \\
0 \\
0
\end{array}\right)=\left(\begin{array}{cccc}
1 & 0 & 0 & 0 \\
-V & 1 & 0 & 0 \\
0 & 0 & 1 & 0 \\
0 & 0 & 0 & 1
\end{array}\right)\left(\begin{array}{c}
m_{B} \\
p_{B} \\
0 \\
0
\end{array}\right),
$$

we obtain the momenta in the center-of-mass system before the collision;

$$
\begin{aligned}
& p_{A}^{*}=p_{A}-m_{A} V=p_{A}-m_{A} \frac{p_{A}+p_{B}}{m_{A}+m_{B}}=+\frac{m_{B} p_{A}-m_{A} p_{B}}{m_{A}+m_{B}}, \\
& p_{B}^{*}=p_{B}-m_{B} V=p_{B}-m_{B} \frac{p_{A}+p_{B}}{m_{A}+m_{B}}=-\frac{m_{B} p_{A}-m_{A} p_{B}}{m_{A}+m_{B}},
\end{aligned}
$$

where, we used Equation (3). It is natural that $p_{A}^{*}+p_{B}^{*}=0$ is fulfilled because of the definition of the center-of-mass system. Then we define a momentum $p^{*}$ as

$$
p^{*} \equiv \frac{m_{B} p_{A}-m_{A} p_{B}}{m_{A}+m_{B}}=p_{A}^{*}=-p_{B}^{*},
$$

for later use. Note that the momentum $p^{*}$ is written in terms of velocity as $p^{*}=\frac{m_{B} p_{A}-m_{A} p_{B}}{m_{A}+m_{B}}=\frac{m_{A} m_{B}}{m_{A}+m_{B}}\left(v_{A}-v_{B}\right)$, which is usually seen in textbooks. The energies of the particles in the system are given by

$$
E_{A}^{*}=\frac{\left(p_{A}^{*}\right)^{2}}{2 m_{A}}=\frac{\left(p^{*}\right)^{2}}{2 m_{A}}=\frac{1}{2 m_{A}}\left(\frac{m_{B} p_{A}-m_{A} p_{B}}{m_{A}+m_{B}}\right)^{2}
$$




$$
E_{B}^{*}=\frac{\left(p_{B}^{*}\right)^{2}}{2 m_{B}}=\frac{\left(p^{*}\right)^{2}}{2 m_{B}}=\frac{1}{2 m_{B}}\left(\frac{m_{B} p_{A}-m_{A} p_{B}}{m_{A}+m_{B}}\right)^{2} .
$$

\section{Energies in the laboratory system before the collision}

Before concluding this subsection, we write down the energies in the laboratory system before the collision in terms of $p^{*}$. From Equations (5) and (6), we obtain

$$
p_{A}=m_{A} V+p^{*}, \quad p_{B}=m_{B} V-p^{*} .
$$

Thus, the kinetic energies in the laboratory system before the collision are given by

$$
\begin{aligned}
& E_{A}=\frac{\left(p_{A}\right)^{2}}{2 m_{A}}=\frac{m_{A}}{2} V^{2}+V p^{*}+\frac{\left(p^{*}\right)^{2}}{2 m_{A}}, \\
& E_{B}=\frac{\left(p_{B}\right)^{2}}{2 m_{B}}=\frac{m_{B}}{2} V^{2}-V p^{*}+\frac{\left(p^{*}\right)^{2}}{2 m_{B}},
\end{aligned}
$$

and their sum becomes

$$
E_{A}+E_{B}=\frac{m_{A}+m_{B}}{2} V^{2}+\frac{\left(p^{*}\right)^{2}}{2}\left(\frac{1}{m_{A}}+\frac{1}{m_{B}}\right)=\frac{\left(p_{A}+p_{B}\right)^{2}}{2\left(m_{A}+m_{B}\right)}+\frac{\left(p^{*}\right)^{2}}{2 \frac{m_{A} m_{B}}{m_{A}+m_{B}}},
$$

where, we used Equation (3). The first term of the right hand side shows the kinetic energy of the center-of-mass, while the second term shows the kinetic energy of the relative motion with respect to the center-of-mass.

\subsection{Momentum in the Center-of-Mass System after the Collision}

We discuss the strategy 3 in the Introduction. We determine the momenta in the center-of-mass system after the collision. In this frame, the particles move in the opposite direction. We introduce the coefficient of restitution $e(0 \leq e \leq 1)$, and write down the momenta in the center-of-mass system after the collision

$$
p_{A}^{\prime *} \equiv-e p_{A}^{*}=-e p^{*}, \quad p_{B}^{\prime *} \equiv-e p_{B}^{*}=+e p^{*} .
$$

Note that these momenta can be derived from the usual definition of the coefficient of restitution: $e=-\frac{v_{A}^{\prime}-v_{B}^{\prime}}{v_{A}-v_{B}}$. In other words, the particles move in the opposite direction after the collision and the magnitudes of the momenta are decreased by $e$. The kinetic energies are obtained by

$$
\begin{aligned}
& E_{A}^{\prime *}=\frac{\left(p_{A}^{\prime *}\right)^{2}}{2 m_{A}}=e^{2} \frac{\left(p^{*}\right)^{2}}{2 m_{A}}=e^{2} E_{A}^{*}, \\
& E_{B}^{\prime *}=\frac{\left(p_{B}^{\prime *}\right)^{2}}{2 m_{B}}=e^{2} \frac{\left(p^{*}\right)^{2}}{2 m_{B}}=e^{2} E_{B}^{*},
\end{aligned}
$$

where, $E_{A}^{*}$ and $E_{B}^{*}$ are given by Equations (8) and (9). Namely, the kinetic energies in the center-of-mass system are decreased by $e^{2}$. 


\subsection{Momentum in the Laboratory System after the Collision}

We discuss the strategy 4 in the Introduction. Consider the Galilean inverse transformation for each particle,

$$
\left(\begin{array}{c}
m_{A} \\
p_{A}^{\prime} \\
0 \\
0
\end{array}\right)=\left(\begin{array}{cccc}
1 & 0 & 0 & 0 \\
V & 1 & 0 & 0 \\
0 & 0 & 1 & 0 \\
0 & 0 & 0 & 1
\end{array}\right)\left(\begin{array}{c}
m_{A} \\
p_{A}^{\prime *} \\
0 \\
0
\end{array}\right), \quad\left(\begin{array}{c}
m_{B} \\
p_{B}^{\prime} \\
0 \\
0
\end{array}\right)=\left(\begin{array}{cccc}
1 & 0 & 0 & 0 \\
V & 1 & 0 & 0 \\
0 & 0 & 1 & 0 \\
0 & 0 & 0 & 1
\end{array}\right)\left(\begin{array}{c}
m_{B} \\
p_{B}^{\prime *} \\
0 \\
0
\end{array}\right),
$$

we obtain the momenta in the laboratory system after the collision. From the second row of these matrices, we obtain

$$
\begin{aligned}
& p_{A}^{\prime}=m_{A} V+p_{A}^{\prime *}=m_{A} V-e p^{*}=p_{A}-(1+e) p^{*}, \\
& p_{B}^{\prime}=m_{B} V+p_{B}^{\prime *}=m_{B} V+e p^{*}=p_{B}+(1+e) p^{*} .
\end{aligned}
$$

The second terms of the right hand side are interpreted by the momentum lost by particle $\mathrm{A}$ and the momentum gained by particle $\mathrm{B}$, which are called impulse in Newtonian mechanics. Adding the two equations, we easily see the conservation of momentum: $p_{A}^{\prime}+p_{B}^{\prime}=p_{A}+p_{B}$. And we also obtain the kinetic energies from Equations (18) and (19),

$$
\begin{aligned}
& E_{A}^{\prime}=\frac{\left(p_{A}^{\prime}\right)^{2}}{2 m_{A}}=\frac{m_{A}}{2} V^{2}-e V p^{*}+e^{2} \frac{\left(p^{*}\right)^{2}}{2 m_{A}}, \\
& E_{B}^{\prime}=\frac{\left(p_{B}^{\prime}\right)^{2}}{2 m_{B}}=\frac{m_{B}}{2} V^{2}+e V p^{*}+e^{2} \frac{\left(p^{*}\right)^{2}}{2 m_{B}} .
\end{aligned}
$$

Summing these kinetic energies, we obtain the total energies after the collision

$$
E_{A}^{\prime}+E_{B}^{\prime}=\frac{\left(p_{A}+p_{B}\right)^{2}}{2\left(m_{A}+m_{B}\right)}+e^{2} \frac{\left(p^{*}\right)^{2}}{2 \frac{m_{A} m_{B}}{m_{A}+m_{B}}} .
$$

Comparing this equation with Equation (13), we understand the followings: the first term, which is the kinetic energy of the center-of-mass, does not change, while the second term, which is the kinetic energy of the relative motion with respect to the center-of-mass, decreases by $e^{2}$. By using Equation (13), we obtain

$$
E_{A}+E_{B}=E_{A}^{\prime}+E_{B}^{\prime}+\left(1-e^{2}\right) \frac{\left(p^{*}\right)^{2}}{2 \frac{m_{A} m_{B}}{m_{A}+m_{B}}} .
$$

The third term of the right hand side is called $Q$-value, which is larger than zero. Namely, the total energy of the whole system is decreased in $Q$-value.

\subsection{In Case of $p_{B}=0$}

We discuss the strategy 5 in the Introduction. In this case, we clearly see the relation 


$$
p^{*}=m_{B} V=\frac{m_{B} p_{A}}{m_{A}+m_{B}},
$$

and obtain the momenta in the laboratory system after the collision

$$
\begin{gathered}
p_{A}^{\prime}=p_{A}-(1+e) p^{*}=\frac{m_{A}-e m_{B}}{m_{A}+m_{B}} p_{A}, \\
p_{B}^{\prime}=+(1+e) p^{*}=\frac{m_{B}+e m_{B}}{m_{A}+m_{B}} p_{A} .
\end{gathered}
$$

We obviously understand the conservation of the momentum: $p_{A}^{\prime}+p_{B}^{\prime}=p_{A}$. In addition, we obtain the kinetic energies for each particle

$$
\begin{gathered}
E_{A}^{\prime}=\frac{\left(p_{A}^{\prime}\right)^{2}}{2 m_{A}}=\left(\frac{m_{A}-e m_{B}}{m_{A}+m_{B}}\right)^{2} E_{A}, \\
E_{B}^{\prime}=\frac{\left(p_{B}^{\prime}\right)^{2}}{2 m_{B}}=(1+e)^{2} \frac{m_{A} m_{B}}{\left(m_{A}+m_{B}\right)^{2}} E_{A},
\end{gathered}
$$

and energy conservation law

$$
E_{A}=E_{A}^{\prime}+E_{B}^{\prime}+\left(1-e^{2}\right) \frac{m_{B}}{m_{A}+m_{B}} E_{A} .
$$

\subsection{In Case of $p_{B}=0$ and $m_{A}=m_{B}=m$}

We discuss the strategy 6 in the Introduction. We easily see the relation

$$
p^{*}=m_{A} V=m_{B} V=\frac{p_{A}}{2},
$$

and obtain the momenta in the laboratory system after the collision

$$
p_{A}^{\prime}=\frac{1-e}{2} p_{A}, \quad p_{B}^{\prime}=\frac{1+e}{2} p_{A} .
$$

We also obtain the kinetic energies

$$
E_{A}^{\prime}=\frac{(1-e)^{2}}{4} E_{A}, \quad E_{B}^{\prime}=\frac{(1+e)^{2}}{4} E_{A},
$$

and their sum

$$
E_{A}=E_{A}^{\prime}+E_{B}^{\prime}+\frac{1-e^{2}}{2} E_{A}
$$

Here, $e=1$ shows the elastic collisions. After the collision, the incident particle A stops and the initially rest particle B moves with the momentum of which the particle A had before the collision.

\section{Elastic Collisions in Two Dimensions}

Let us turn our discussion to the case of the two dimensional elastic collisions, i.e., the case in which the coefficient of restitution $e$ is equal to 1 .

We suppose that the motions of the particles are restricted in the $x$ - $y$ plain so that the $z$-component of the momentum is zero. Since the motions of the par- 
ticles are supposed along the $x$-direction before the collision, we trace the same discussion of subsections 2.1 and 2.2. So, let us start from the strategy 3 in the Introduction.

The diagrammatic technique of this section is fully discussed in [6].

\subsection{Momentum in the Center-of-Mass System after the Collision}

We discuss the strategy 3 in the Introduction. In the center-of-mass system, the magnitudes of the momenta do not change before and after the collision. Thus, we write down the momenta in the same way with Equation (7),

$$
p^{*} \equiv \frac{m_{B} p_{A}-m_{A} p_{B}}{m_{A}+m_{B}}=p_{A}^{*}=p_{B}^{*}=p_{A}^{\prime *}=p_{B}^{\prime *} .
$$

However, the direction of the momenta changes in the two dimensional collision. As shown in Figure 2, we define the sense of the momentum $\boldsymbol{p}_{A}^{\prime *}$ as $\boldsymbol{n}^{*}=\left(\cos \theta^{*}, \sin \theta^{*}\right)$, where $\theta^{*}$ is the scattering angle of the particle $\mathrm{A}$ in the center-of-mass system. In other words, the momenta after the collisions are denoted by the vector-form in two dimensions

$$
\boldsymbol{p}_{A}^{\prime *}=p^{*} \boldsymbol{n}^{*}=-\boldsymbol{p}_{B}^{\prime *} .
$$

Since the magnitudes of the momenta do not change in this frame after the collision, the kinetic energies of each particle do not change:

$$
\begin{aligned}
& E_{A}^{\prime *}=\frac{\left(p_{A}^{\prime *}\right)^{2}}{2 m_{A}}=\frac{\left(p^{*}\right)^{2}}{2 m_{A}}=E_{A}^{*}, \\
& E_{B}^{\prime *}=\frac{\left(p_{B}^{\prime *}\right)^{2}}{2 m_{B}}=\frac{\left(p^{*}\right)^{2}}{2 m_{B}}=E_{B}^{*},
\end{aligned}
$$

where, $E_{A}^{*}$ and $E_{B}^{*}$ are the same with Equations (8) and (9).

\subsection{Momentum in the Laboratory System after the Collision}

We discuss the strategy 4 in the Introduction. The motion of the particles after the collision is supposed to occur in the $x$ - $y$ plain. Thus, the momenta are written by $\boldsymbol{p}_{A}^{\prime}=\left(p_{A x}^{\prime}, p_{A y}^{\prime}, 0\right)=\left(p_{A}^{\prime} \cos \theta, p_{A}^{\prime} \sin \theta, 0\right)$ and $\boldsymbol{p}_{B}^{\prime}=\left(p_{B x}^{\prime}, p_{B y}^{\prime}, 0\right)=\left(p_{B}^{\prime} \cos \phi,-p_{B}^{\prime} \sin \phi, 0\right)$, where $\theta$ and $\phi$ are the scattering angle of the particles A and B in the laboratory system as shown in Figure 2.

From the Galilean inverse transformation, we obtain the momenta in the laboratory system after the collision by using Equation (35)

$$
\begin{aligned}
& \left(\begin{array}{c}
m_{A} \\
p_{A x}^{\prime} \\
p_{A y}^{\prime} \\
0
\end{array}\right)=\left(\begin{array}{c}
m_{A} \\
p_{A}^{\prime} \cos \theta \\
p_{A}^{\prime} \sin \theta \\
0
\end{array}\right)=\left(\begin{array}{llll}
1 & 0 & 0 & 0 \\
V & 1 & 0 & 0 \\
0 & 0 & 1 & 0 \\
0 & 0 & 0 & 1
\end{array}\right)\left(\begin{array}{c}
m_{A} \\
p^{*} \cos \theta^{*} \\
p^{*} \sin \theta^{*} \\
0
\end{array}\right), \\
& \left(\begin{array}{c}
m_{B} \\
p_{B x}^{\prime} \\
p_{B y}^{\prime} \\
0
\end{array}\right)=\left(\begin{array}{c}
m_{B} \\
p_{B}^{\prime} \cos \phi \\
-p_{B}^{\prime} \sin \phi \\
0
\end{array}\right)=\left(\begin{array}{llll}
1 & 0 & 0 & 0 \\
V & 1 & 0 & 0 \\
0 & 0 & 1 & 0 \\
0 & 0 & 0 & 1
\end{array}\right)\left(\begin{array}{c}
m_{B} \\
-p^{*} \cos \theta^{*} \\
-p^{*} \sin \theta^{*} \\
0
\end{array}\right) .
\end{aligned}
$$



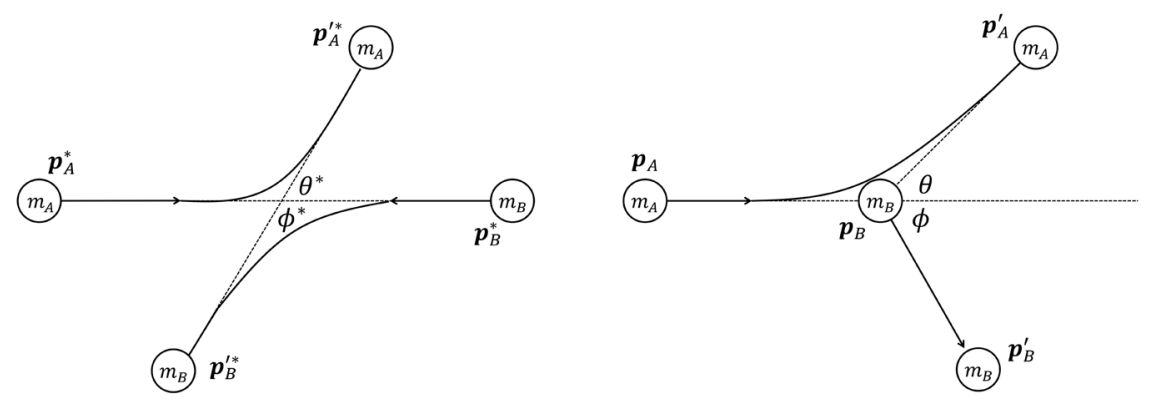

Figure 2. Left: The collision in the center-of-mass system. The scattering angles $\theta^{*}$ and $\phi^{*}$ have the relation $\theta^{*}+\phi^{*}=\pi$. Right: The collision in the laboratory system.

From the second and third row of these matrices, we obtain $x$ and $y$ components of the momentum of the particle $\mathrm{A}$,

$$
\begin{gathered}
p_{A x}^{\prime}=p_{A}^{\prime} \cos \theta=m_{A} V+p^{*} \cos \theta^{*}, \\
p_{A y}^{\prime}=p_{A}^{\prime} \sin \theta=p^{*} \sin \theta^{*},
\end{gathered}
$$

and of the particle B,

$$
\begin{gathered}
p_{B x}^{\prime}=p_{B}^{\prime} \cos \phi=m_{B} V-p^{*} \cos \theta^{*}, \\
p_{B y}^{\prime}=-p_{B}^{\prime} \sin \phi=-p^{*} \sin \theta^{*} .
\end{gathered}
$$

Combining with the relation $\cos ^{2} \theta^{*}+\sin ^{2} \theta^{*}=1$, we obtain

$$
\begin{aligned}
& \left(\frac{p_{A x}^{\prime}-m_{A} V}{p^{*}}\right)^{2}+\left(\frac{p_{A y}^{\prime}}{p^{*}}\right)^{2}=1, \\
& \left(\frac{p_{B x}^{\prime}-m_{B} V}{p^{*}}\right)^{2}+\left(\frac{p_{B y}^{\prime}}{p^{*}}\right)^{2}=1 .
\end{aligned}
$$

These equations show the circle with the radius $p^{*}$ and the center $\left(m_{A} V, 0,0\right)$ and $\left(m_{B} V, 0,0\right)$. The circle in Equation (44) of the particle $\mathrm{A}$ is drawn in the literature [4] [6].

The kinetic energies of each particle are obtained by using Equations (40), (41) and Equations (42), (43)

$$
\begin{aligned}
& E_{A}^{\prime}=\frac{\left(p_{A}^{\prime}\right)^{2}}{2 m_{A}}=\frac{m_{A}}{2} V^{2}+V p^{*} \cos \theta^{*}+\frac{\left(p^{*}\right)^{2}}{2 m_{A}}, \\
& E_{B}^{\prime}=\frac{\left(p_{B}^{\prime}\right)^{2}}{2 m_{B}}=\frac{m_{B}}{2} V^{2}-V p^{*} \cos \theta^{*}+\frac{\left(p^{*}\right)^{2}}{2 m_{B}} .
\end{aligned}
$$

Since their sum is the same with Equation (13), we obtain the conservation of energies:

$$
E_{A}^{\prime}+E_{B}^{\prime}=E_{A}+E_{B}
$$

\subsection{In Case of $p_{B}=0$}

We discuss the strategy 5 in the Introduction. The same condition of Equation 
(24) is satisfied. Dividing two equations of Equations (40), (41) and Equations (42), (43), we obtain the relations of the scattering angles

$$
\begin{gathered}
\tan \theta=\frac{\sin \theta^{*}}{\frac{m_{A}}{m_{B}}+\cos \theta^{*}}, \\
\tan \phi=\frac{\sin \theta^{*}}{1-\cos \theta^{*}}=\frac{\sin \phi^{*}}{1+\cos \phi^{*}},
\end{gathered}
$$

where, we used $\theta^{*}+\phi^{*}=\pi$. Using Equations (40), (41) and Equations (42), (43) with (49), we obtain the momenta

$$
\begin{gathered}
p_{A}^{\prime}=\frac{p_{A}}{m_{A}+m_{B}} \sqrt{m_{A}^{2}+m_{B}^{2}+2 m_{A} m_{B} \cos \theta^{*}}, \\
p_{B}^{\prime}=\frac{2 m_{B} p_{A}}{m_{A}+m_{B}} \sin \left(\frac{\theta^{*}}{2}\right),
\end{gathered}
$$

and the energies

$$
\begin{aligned}
& E_{A}^{\prime}=\frac{\left(p_{A}^{\prime}\right)^{2}}{2 m_{A}}=E_{A} \times \frac{m_{A}^{2}+m_{B}^{2}+2 m_{A} m_{B} \cos \theta^{*}}{\left(m_{A}+m_{B}\right)^{2}}, \\
& E_{B}^{\prime}=\frac{\left(p_{B}^{\prime}\right)^{2}}{2 m_{B}}=E_{A} \times \frac{4 m_{A} m_{B}}{\left(m_{A}+m_{B}\right)^{2}} \sin ^{2}\left(\frac{\theta^{*}}{2}\right),
\end{aligned}
$$

after the collision.

\subsection{In Case of $p_{B}=0$ and $m_{A}=m_{B}=m$}

We discuss the strategy 6 in the Introduction. The same condition of Equation (30) is satisfied. We obtain the momenta from Equations (51) and (52)

$$
p_{A}^{\prime}=p_{A} \cos \left(\frac{\theta^{*}}{2}\right), \quad p_{B}^{\prime}=p_{A} \sin \left(\frac{\theta^{*}}{2}\right)
$$

and the energies from Equations (53) and (54)

$$
E_{A}^{\prime}=E_{A} \cos ^{2}\left(\frac{\theta^{*}}{2}\right), \quad E_{B}^{\prime}=E_{A} \sin ^{2}\left(\frac{\theta^{*}}{2}\right) .
$$

The product of the relation of the angles, Equations (49) and (50), becomes

$$
\tan \theta \times \tan \phi=\frac{\sin \theta^{*}}{1+\cos \theta^{*}} \frac{\sin \theta^{*}}{1-\cos \theta^{*}}=1 .
$$

Since the addition theorem for tangent

$$
\tan (\theta+\phi)=\frac{\tan \theta+\tan \phi}{1-\tan \theta \tan \phi}=\infty,
$$

we obtain the relation in this case

$$
\theta+\phi=\frac{\pi}{2},
$$

which is well-known in the textbooks of physics. 


\section{Summary}

We reexamined the collision problems in Newtonian mechanics. The usual treatment of the introductory textbook of physics is that we have to solve the simultaneous equations for obtaining the final states. In contrast to this, we introduce the mass-momentum "vectors", though this has no nature of the vector, and show the validity of them. Just moving the laboratory and center-of-mass systems to and fro by the Galilean transformation, we obtain the same results which are given by solving the simultaneous equations. This process is applicable to both one and two dimensions in the same fashion. This gives students the unified way for seeing the collision problems.

Illustrating the collision problems is rewarding to understand them. For one dimensional collision, the mass-momentum "vectors" play the central role [2] [3]. For two dimensional collisions, the circles on the momentum space are important [4] [6].

This discussion in this article is also applicable to special relativistic collision problems in the same fashion [7]. In this case too, we do not have to solve the simultaneous equations any more. Furthermore, all results in this paper are obtained from the relativistic collisions by the limit of $c \rightarrow \infty$ [7] [8], where $c$ is the speed of light.

\section{Acknowledgements}

The author thanks the anonymous reviewer for his helpful suggestions.

\section{Conflicts of Interest}

The author declares no conflicts of interest regarding the publication of this paper.

\section{References}

[1] Halpern, A. (1995) Beginning Physics I. McGraw-Hill, New York.

[2] Takeuchi, T. (2010) An Illustrated Guide to Relativity. Cambridge University Press, Cambridge. https://doi.org/10.1017/CBO9780511779121

[3] Ogura, A. (2017) Analyzing Collisions in Classical Mechanics Using Mass-Momentum Diagrams. European Journal of Physics, 38, No. 5.

https://doi.org/10.1088/1361-6404/aa750b

[4] Landau, L.D. and Lifshitz, E.M. (1976) Mechanics. Butterworth-Heinenann.

[5] Bokor, N. (2011) Analyzing Collisions Using Minkowski Diagrams in Momentum Space. European Journal of Physics, 32, 773-782.

https://doi.org/10.1088/0143-0807/32/3/013

[6] Ogura, A. (2018) Diagrammatic Approach for Investigating Two Dimensional Elastic Collisions in Momentum Space I: Newtonian Mechanics. World Journal of $\mathrm{Me}$ chanics, 8, 343-352. https://doi.org/10.4236/wjm.2018.89025

[7] Ogura, A. (2019) Elastic Collisions in Minkowski Momentum Space with Lorentz Transformations. World Journal of Mechanics, 9, 267-284.

https://doi.org/10.4236/wjm.2019.912018 
[8] Ogura, A. (2018) Diagrammatic Approach for Investigating Two Dimensional Elastic Collisions in Momentum Space II: Special Relativity. World Journal of Mechanics, 8, 353-361. https://doi.org/10.4236/wjm.2018.89026 